\title{
An Algorithm for Traffic Equilibrium Flow with Capacity Constraints of Arcs
}

\author{
Zhi Lin \\ College of Sciences, Chongqing Jiaotong University, Chongqing, China \\ Email: linzhi7525@163.com
}

Received 22 July 2015; accepted 19 October 2015; published 22 October 2015

Copyright (C) 2015 by author and Scientific Research Publishing Inc. This work is licensed under the Creative Commons Attribution International License (CC BY). http://creativecommons.org/licenses/by/4.0/

\begin{abstract}
In the traffic equilibrium problem, we introduce capacity constraints of arcs, extend Beckmann's formula to include these constraints, and give an algorithm for traffic equilibrium flows with capacity constraints on arcs. Using an example, we illustrate the application of the algorithm and show that Beckmann's formula is a sufficient condition only, not a necessary condition, for traffic equilibrium with capacity constraints of arcs.
\end{abstract}

\section{Keywords}

The Traffic Equilibrium Problem with Capacity Constraints of Arcs, Equilibrium Flow, Algorithm, Capacity of Arc, Saturated Path

\section{Introduction}

In [1], Wardrop introduced the traffic equilibrium problem and proposed a scalar equilibrium principle. In [2], Beckmann et al. gave a mathematical programming problem that was equivalent to Wardrop's traffic equilibrium problem. Using Beckmann's work, it is possible to find the traffic equilibrium flow if the cost function is a scalar. In [3], Chen and Yen generalized Wardrop's equilibrium principle to a (weak) vector equilibrium principle. In [4] [5], we extended the vector equilibrium principle to capacity constraints along arcs and derived existence and stability results for (weak) vector equilibrium flows. In this paper, we introduce the traffic equilibrium problem with capacity constraints of arcs (TEPCCA), extend Beckmann's transformation to cover capacity constraints along arcs, and give an algorithm for traffic equilibrium flows with capacity constraints of arcs for scalar cost functions. As an example, we illustrate the algorithm and show that Beckmann's transformation is a sufficient condition only, not a necessary condition, for traffic equilibria with capacity constraints of arcs. For other results with respect to traffic equilibrium with capacity constraints of arcs, we refer to [6], and for other results with respect to algorithms of equilibrium flows, we refer to [7]-[9] and the references therein. 
For a traffic network, let $V$ denote the set of nodes, $E$ the set of directed arcs, and $W$ the set of origindestination O-D pairs. For each $\omega \in W$, let $P_{\omega}$ denote the set of available paths joining O-D pair $\omega$ and denote by $K=\bigcup_{\omega \in W} P_{\omega}, m=|K|$. Let $D=\left(d_{\omega}\right)_{\omega \in W}$ denote the demand vector, with $d_{\omega}(>0)$ denoting the traffic demand on O-D pair $\omega$. For each $a \in E$, the arc flow $f_{a} \in R_{+}=\{z \in R: z \geq 0\}$. For each $\omega \in W$ and $k \in P_{\omega}$, let $f_{k}(\geq 0)$ denote the traffic flow on path $k . f=\left(f_{k}\right)_{k \in K}^{\mathrm{T}} \in R_{+}^{m}=\left\{\left(z_{1}, z_{2}, \cdots, z_{m}\right) \in R^{m}: z_{i} \geq 0, i=1,2, \cdots, m\right\}$ is said to be a path flow (flow). Clearly, for $a \in E, f_{a}=\sum_{\omega \in W} \sum_{k \in P_{\omega}} \delta_{a k} f_{k}$, where $\delta_{a k}=1$ if arc $a$ belongs to path $k$, otherwise $\delta_{a k}=0$, thus $f_{a}=f_{a}(f)$. Let $C=\left(c_{a}\right)_{a \in E}$ denote the capacity vector, where $c_{a}(>0)$ denotes the capacity of flow on arc $a$. A traffic network is usually denoted by $\aleph=\{V, E, W, D, C\}$. For each arc $a \in E$, the flow on arc $a$ needs to satisfy the capacity constraint: $c_{a} \geq f_{a} \geq 0$, and for each $\omega \in W$, the flow $f$ needs to satisfy the demand constraint: $\sum_{k \in P} f_{k}=d_{\omega}$. A flow $f$ satisfying the demand and capacity constraints is called a feasible path flow (a feasible flow for short). Let

$$
A=\left\{f \in R_{+}^{m}: \forall \omega \in W, \sum_{k \in P_{\omega}} f_{k}=d_{\omega} \text { and } \forall a \in E, c_{a} \geq f_{a} \geq 0\right\} .
$$

In this paper, we assume that for each $\omega \in W$, the demand $d_{\omega}$ is fixed and $A \neq \varnothing$. It is easy to verify that $A$ is convex and compact. For each $a \in E$, let $t_{a}=t_{a}\left(f_{a}\right)=t_{a}(f) \in R_{+}$be the cost on arc $a$, and for each $\omega \in W, k \in P_{w}$, the cost $t_{k}$ along path $k$ is assumed to be the sum of all arc costs along $k$, i.e., $t_{k}(f)=\sum_{a \in E} \delta_{a k} t_{a}(f)$.

\section{Preliminaries}

For the following definitions, see [4] [5].

Definition 2.1. Assume that a flow $f \in A$.

1) for $a \in E$, if $f_{a}=c_{a}$, then $a$ is said to be a saturated $\operatorname{arc}$ of flow $f$, otherwise a nonsaturated arc of flow $f$.

2) for $k \in \bigcup_{\omega \in W} P_{\omega}$, if there exists a saturated arc $a$ of flow $f$ such that $a$ belongs to path $k$, then $k$ is said to be a saturated path of flow $f$, otherwise a nonsaturated path of flow $f$.

Definition 2.2. (Equilibrium principle with capacity constraints of arcs). A flow $f \in A$ is said to be in equilibrium if,

$$
\begin{aligned}
& \forall \omega \in W, \forall k, j \in P_{\omega}, t_{k}(f)-t_{j}(f)>0 \\
& \Rightarrow f_{k}=0 \text { or path } j \text { is a saturated path of flow } f .
\end{aligned}
$$

$f$ is said to be an equilibrium flow or solution of the TEPCCA. A TEPCCA is usually denoted by $\Gamma=\{\aleph, A, t\}$.

\section{A Generalization of Beckmann’s Formula}

For the TEPCCA $\Gamma=\{\aleph, A, t\}$, construct the following mathematical programming problem $Q$ :

$$
\begin{aligned}
& \operatorname{Min} z(f)=\sum_{a \in E} \int_{0}^{f_{a}} t_{a}(x) \mathrm{d} x \\
& \text { s.t. } \begin{cases}\sum_{k} f_{k}=d_{\omega}, & \forall \omega \in W, k \in P_{\omega} \\
f_{a}=\sum_{\omega} \sum_{k} f_{k} \delta_{a k} \leq c_{a}, & \forall a \in E, \omega \in W, k \in P_{\omega} \\
f_{k} \geq 0, & \forall \omega \in W, k \in P_{\omega} .\end{cases}
\end{aligned}
$$

The above formula is a generalization of Beckmann's formula. The next theorem shows that each solution of the generalization of Beckmann's formula is an equilibrium flow for $\Gamma$.

Theorem 3.1. Consider the TEPCCA. Assume that for each $a \in E, t_{a}(f)$ is continuous on $R_{+}^{m}$, then the flow $f \in A$ is in equilibrium if $f$ solves the mathematical programming problem $Q$.

Proof. Set $h_{\omega}=\sum_{k} f_{k}-d_{\omega}$ and $g_{a}=c_{a}-f_{a}$. The Kuhn-Tucker conditions for the problem $Q$ are: 


$$
\begin{cases}\frac{\partial z[f]}{\partial f_{k}}-\sum_{\omega} \rho_{\omega} \frac{\partial h_{\omega}}{\partial f_{k}}-\sum_{a} \lambda_{a} \frac{\partial g_{a}}{\partial f_{k}}-\beta_{k}=0, & \forall \omega \in W, k \in P_{\omega} \\ \lambda_{a}\left(c_{a}-f_{a}\right)=0, & \forall a \in E \\ \beta_{k} f_{k} \geq 0, & \forall \omega \in W, k \in P_{\omega} \\ \rho_{\omega} \geq 0, \lambda_{a} \geq 0, \beta_{k} \geq 0, & \forall \omega \in W, a \in E, k \in P_{\omega}\end{cases}
$$

where $\rho_{\omega}, \lambda_{a}$ and $\beta_{k}$ are Lagrange multipliers. Since for each $a \in E, t_{a}(f)$ is continuous on $R_{+}^{m}$, we have

$$
\frac{\partial z[f]}{\partial f_{k}}=\frac{\partial}{\partial f_{k}}\left(\sum_{a} \int_{0}^{f_{a}} t_{a}(x) \mathrm{d} x\right)=\sum_{a} \frac{\partial}{\partial f_{a}} \int_{0}^{f_{a}} t_{a}(x) \mathrm{d} x \cdot \frac{\partial f_{a}}{\partial f_{k}}=\sum_{a} t_{a}(f) \delta_{a k}=t_{k}, \sum_{\omega} \rho_{\omega} \frac{\partial h_{\omega}}{\partial f_{k}}=\rho_{\omega} .
$$

When path $k$ is a nonsaturated path of flow $f$, for each $a \in k$, we have $c_{a}-f_{a}>0$. Note that $\lambda_{a}\left(c_{a}-f_{a}\right)=0$, we have $\lambda_{a}=0$. Thus,

$$
\sum_{a} \lambda_{a} \frac{\partial g_{a}}{\partial f_{k}}=\sum_{a \in k} \lambda_{a} \frac{\partial g_{a}}{\partial f_{k}}=-\sum_{a \in k} \lambda_{a} \begin{cases}=0 & \text { if path } k \text { is a nonsaturated path of flow } f \\ \leq 0 & \text { otherwise. }\end{cases}
$$

Hence, when $k$ is a nonsaturated path, we have $f_{k}\left(t_{k}-\rho_{\omega}\right)=0$, i.e.,

$$
\begin{array}{ll}
\text { if } f_{k}>0, & t_{k}=\rho_{\omega} \quad \forall \omega \in W, k \in P_{\omega} \\
\text { if } f_{k}=0, & t_{k} \geq \rho_{\omega} \quad \forall \omega \in W, k \in P_{\omega}
\end{array}
$$

and when $k$ is a saturated path, we have $f_{k}\left(t_{k}-\rho_{\omega}+\sum_{a \in k} \lambda_{a}\right)=0$, i.e.,

$$
\begin{array}{lll}
\text { if } f_{k}>0, \quad t_{k}(\geq 0)=\rho_{\omega}-\sum_{a \in k} \lambda_{a} \leq \rho_{\omega} & \forall \omega \in W, k \in P_{\omega} \\
\text { if } f_{k}=0, \quad t_{k} \geq 0 & \forall \omega \in W, k \in P_{\omega}
\end{array}
$$

In other words, if paths $k$ is a nonsaturated path, then $t_{k} \geq \rho_{\omega}$, and if paths $k$ such that $f_{k}>0$, then $t_{k} \leq \rho_{\omega}$. Thus, for $\forall \omega \in W, \forall k, j \in P_{\omega}, t_{k}(f)-t_{j}(f)>0$ and $j$ is a nonsaturated path, then $f_{k}=0$, otherwise $f_{k}>0$, which implies that $t_{k}(f) \leq \rho_{\omega} \leq t_{j}(f)$, a contradiction. By Definition 2.2, the proof is finished.

From the generalization of Beckmann's formula, it is easy to construct an algorithm to calculate the equilibrium flow for the TEPCCA $\Gamma=\{\aleph, A, t\}$.

\section{An Algorithm for the Traffic Equilibrium Flow with Capacity Constraints of Arcs}

For the TEPCCA $\Gamma=\{\aleph, A, t\}$, because there are usually many paths in $K=\bigcup_{\omega \in W} P_{\omega}$, implying that there are many variable in the generalization of Beckmann's formula, it is often difficult to compute its solution. Note that there are many paths for which the flow is zero in an equilibrium flow. If we delete these from $K$, it does not cause any change in the equilibrium flow. For this season, we construct the following algorithm to compute the equilibrium flow with capacity constraints of arcs. Assume that for each $a \in E, t_{a}(f)$ is continuous on $R_{+}^{m}$.

Step 1. Find a feasible flow $f^{0} \in A$ and denote by $H^{0}=\left\{l \in K: f_{l}^{0}>0\right\}$. Let $i=0$.

Step 2. Solve the restricted problem $\bar{Q}^{i}$ :

$$
\begin{aligned}
& \operatorname{Min} z(f)=\sum_{a \in E} \int_{0}^{f_{a}} t_{a}(x) \mathrm{d} x \\
& \text { s.t. } \begin{cases}\sum_{k} f_{k}=d_{\omega}, & \forall \omega \in W, k \in H^{i} \\
f_{a}=\sum_{\omega} \sum_{k} f_{k} \delta_{a k} \leq c_{a}, & \forall a \in E \\
f_{k} \geq 0, & \forall k \in H^{i} .\end{cases}
\end{aligned}
$$

We obtain solution $f^{i+1} \in A$. For each O-D pair $\omega \in W$, denote by $\tau_{\omega}^{i+1}=\max _{l \in P_{\omega}}\left\{t_{l}\left(f^{i+1}\right): f_{l}^{i+1}>0\right\}$, where $t_{l}\left(f^{i+1}\right)$ denotes the cost of path $l$ when flow is $f^{i+1}$ on the network $\aleph$.

Step 3. After deleting all saturated arcs of the flow $f^{i+1}$ in the network $\aleph$, we compute its shortest path for 
each O-D pair. For each O-D pair $\omega \in W$, let $S_{\omega}^{i+1}=\left\{l \in P_{\omega}: l\right.$ is a shortest path for $\omega$ and $\left.t_{l}\left(f^{i+1}\right)<\tau_{\omega}^{i+1}\right\}$.

Step 4. If $S^{i+1}=\bigcup_{\omega \in W} S_{\omega}^{i+1}=\varnothing$, go to Step 5; otherwise let $H^{i+1}=H^{i} \cup S^{i+1}, i=i+1$ and go to Step 2.

Step 5. The equilibrium flow is $f^{i+1}$ for the TEPCCA and stop.

The following example shows the calculation process of the algorithm.

Example 4.1. Consider the TEPCCA (see Figure 1), where

$$
\begin{aligned}
& V=\{1,2,3,4,5,6\}, \quad E=\left\{e_{1}, e_{2}, e_{3}, e_{4}, e_{5}, e_{6}, e_{7}, e_{8}, e_{9}, e_{10}, e_{11}\right\}, \quad C=(6,7,10,8,7,5,9,7,9,11,7), \\
& W=\{\omega 1, \omega 2\}=\{(1,4),(3,6)\}, \quad D=\left(d_{\omega 1}, d_{\omega 2}\right)=(9,8),
\end{aligned}
$$

and

$$
\begin{aligned}
& t_{e_{1}}\left(f_{e_{1}}\right)=4 f_{e_{1}}^{2}+17, t_{e_{2}}\left(f_{e_{2}}\right)=3 f_{e_{2}}^{2}+18, t_{e_{3}}\left(f_{e_{3}}\right)=30 f_{e_{3}}^{2}+120, t_{e_{4}}\left(f_{e_{4}}\right)=2 f_{e_{4}}^{2}+84, \\
& t_{e_{5}}\left(f_{e_{5}}\right)=f_{e_{5}}^{2}+112, t_{e_{6}}\left(f_{e_{6}}\right)=2 f_{e_{6}}+18, t_{e_{7}}\left(f_{e_{7}}\right)=8 f_{e_{7}}^{2}+62, t_{e_{8}}\left(f_{e_{8}}\right)=6 f_{e_{8}}^{2}+65 \\
& t_{e_{9}}\left(f_{e_{9}}\right)=f_{e_{9}}^{2}+18, t_{e_{10}}\left(f_{e_{10}}\right)=f_{e_{10}}^{2}+15, t_{e_{11}}\left(f_{e_{11}}\right)=2 f_{e_{11}}^{2}+10 .
\end{aligned}
$$

For O-D pair $\omega 1=(1,4): P_{\omega 1}$ contains paths $l_{1}=\left(e_{3}\right), l_{2}=\left(e_{4} e_{10}\right), l_{3}=\left(e_{1} e_{5}\right)$, and $l_{4}=\left(e_{1} e_{6} e_{10}\right)$, and for O-D pair $\omega 2=(3,6): P_{\omega 2}$ contains paths $l_{5}=\left(e_{9}\right), l_{6}=\left(e_{7} e_{11}\right), l_{7}=\left(e_{2} e_{8}\right)$ and $l_{8}=\left(e_{2} e_{6} e_{11}\right)$.

Let $f=\left(f_{1}, f_{2}, f_{3}, f_{4}, f_{5}, f_{6}, f_{7}, f_{8}\right)^{\mathrm{T}} \in R_{+}^{8}$, where $f_{j}$ denotes the flow on path $l_{j}(j=1,2,3,4,5,6,7,8)$. Thus, we have

$$
\begin{aligned}
& f_{e_{1}}=f_{3}+f_{4}, f_{e_{2}}=f_{7}+f_{8}, f_{e_{3}}=f_{1}, f_{e_{4}}=f_{2}, f_{e_{5}}=f_{3}, f_{e_{6}}=f_{4}+f_{8}, \\
& f_{e_{7}}=f_{6}, f_{e_{8}}=f_{7}, f_{e_{9}}=f_{5}, f_{e_{10}}=f_{2}+f_{4}, f_{e_{11}}=f_{6}+f_{8} .
\end{aligned}
$$

Next, we compute the equilibrium flow with capacity constraints of arcs.

1) It is easy to verify that

$$
f^{0}=\left(f_{1}, f_{2}, f_{3}, f_{4}, f_{5}, f_{6}, f_{7}, f_{8}\right)^{\mathrm{T}}=(9,0,0,0,8,0,0,0)^{\mathrm{T}} \in A .
$$

$H^{0}=\left\{l \in K: f_{l}^{0}>0\right\}=\left\{l_{1}, l_{5}\right\}$. Let $i=0$.

2) Solve the restricted problem $\bar{Q}^{0}$ :

$$
\begin{aligned}
& \operatorname{Minz}(f)=\sum_{a \in E} \int_{0}^{f_{a}} t_{a}(x) \mathrm{d} x=10 f_{1}^{3}+120 f_{1}+\frac{1}{3} f_{5}^{3}+18 f_{5} \\
& \text { s.t. }\left\{\begin{array}{l}
f_{1}=9 \\
f_{5}=8
\end{array}\right.
\end{aligned}
$$

We obtain solution $f^{1}=f^{0} \in A$. For O-D pair $\omega 1=(1,4), \tau_{\omega 1}^{1}=2550$, and for O-D pair $\omega 2=(3,6)$, $\tau_{\omega 2}^{1}=82$.

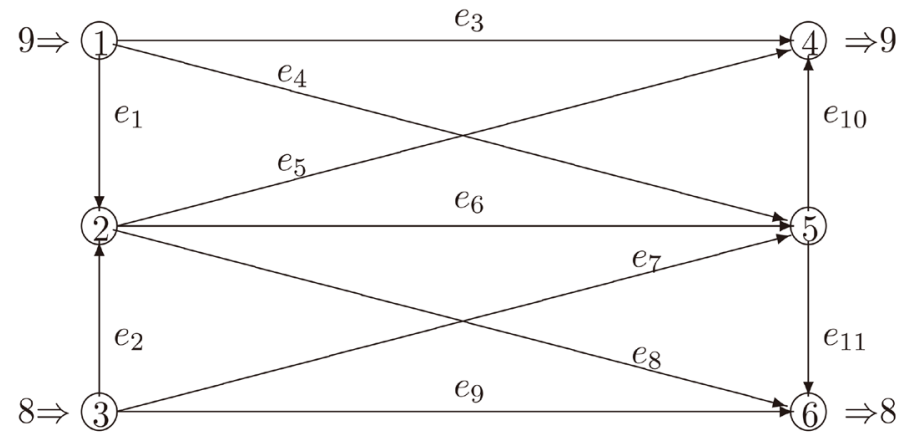

Figure 1. A traffic network. 
3) There is no saturated arc of flow $f^{1}$ in the network $\mathfrak{k}$. For O-D pair $\omega 1=(1,4)$, it is easy to verify that the shortest path is $l_{4}$, whereas for O-D pair $\omega 2=(3,6)$, the shortest path is $l_{8}$. Note that

$$
t_{l_{4}}\left(f^{1}\right)=50<\tau_{\omega 1}^{1}=2550, t_{l_{8}}\left(f^{1}\right)=46<\tau_{\omega 2}^{1}=82,
$$

thus $S_{\omega 1}^{1}=\left\{l_{4}\right\}, S_{\omega 2}^{1}=\left\{l_{8}\right\}$.

4) Since $S^{1}=S_{\omega 1}^{1} \cup S_{\omega 2}^{1}=\left\{l_{4}, l_{8}\right\} \neq \varnothing$, let $H^{1}=H^{0} \cup S^{1}=\left\{l_{1}, l_{4}, l_{5}, l_{8}\right\}$ and solve the restricted problem $\bar{Q}^{1}$ :

$$
\begin{aligned}
& \operatorname{Minz}(f)=\sum_{a \in E} \int_{0}^{f_{a}} t_{a}(x) \mathrm{d} x=10 f_{1}^{3}+120 f_{1}+\frac{4}{3} f_{4}^{3}+17 f_{4}+\left(f_{4}+f_{8}\right)^{2}+18\left(f_{4}+f_{8}\right) \\
& +\frac{1}{3} f_{4}^{3}+15 f_{4}+\frac{1}{3} f_{5}^{3}+18 f_{5}+f_{8}^{3}+18 f_{8}+\frac{2}{3} f_{8}^{3}+10 f_{8} \\
& =10 f_{1}^{3}+120 f_{1}+\frac{5}{3} f_{4}^{3}+50 f_{4}+\left(f_{4}+f_{8}\right)^{2}+\frac{1}{3} f_{5}^{3}+18 f_{5}+\frac{5}{3} f_{8}^{3}+46 f_{8} \\
& \text { s.t. }\left\{\begin{array}{l}
f_{1}+f_{4}=9 \\
f_{5}+f_{8}=8 \\
f_{4}+f_{8} \leq 5 \\
10 \geq
\end{array} f_{1} \geq 0,6 \geq f_{4} \geq 0,9 \geq f_{5} \geq 0,7 \geq f_{8} \geq 0\right.
\end{aligned}
$$

We obtain solution $f^{2}=(4,0,0,5,8,0,0,0)^{\mathrm{T}} \in A$. For O-D pair $\omega 1=(1,4), \tau_{\omega 1}^{2}=600$, and for O-D pair $\omega 2=(3,6), \tau_{\omega 2}^{2}=82$.

5) After deleting saturated arc $e_{6}$ of flow $f^{2}$ in the network $\aleph$. For O-D pair $\omega 1=(1,4)$, it is easy to verify that the shortest path is $l_{2}$, whereas for O-D pair $\omega 2=(3,6)$, the shortest path is $l_{5}$. Note that

$$
t_{l_{2}}\left(f^{2}\right)=124<\tau_{\omega 1}^{2}=600, t_{l_{5}}\left(f^{2}\right)=82=\tau_{\omega 2}^{2}=82,
$$

thus $S_{\omega 1}^{2}=\left\{l_{2}\right\}, S_{\omega 2}^{2}=\varnothing$.

6) Since $S^{2}=S_{\omega 1}^{2} \cup S_{\omega 1}^{2}=\left\{l_{2}\right\} \neq \varnothing$, let $H^{2}=H^{1} \cup S^{2}=\left\{l_{1}, l_{2}, l_{4}, l_{5}, l_{8}\right\}$ and solve the restricted problem $\bar{Q}^{2}$ :

$$
\begin{aligned}
& \operatorname{Min} z(f)=\sum_{a \in E} \int_{0}^{f_{a}} t_{a}(x) \mathrm{d} x=10 f_{1}^{3}+120 f_{1}+\frac{4}{3} f_{4}^{3}+17 f_{4}+\left(f_{4}+f_{8}\right)^{2}+18\left(f_{4}+f_{8}\right) \\
& +\frac{1}{3}\left(f_{2}+f_{4}\right)^{3}+15\left(f_{2}+f_{4}\right)+\frac{2}{3} f_{2}^{3}+84 f_{2}+\frac{1}{3} f_{5}^{3}+18 f_{5}+f_{8}^{3}+18 f_{8}+\frac{2}{3} f_{8}^{3}+10 f_{8} \\
& =10 f_{1}^{3}+120 f_{1}+\frac{4}{3} f_{4}^{3}+50 f_{4}+\left(f_{4}+f_{8}\right)^{2}+\frac{1}{3}\left(f_{2}+f_{4}\right)^{3} \\
& +\frac{2}{3} f_{2}^{3}+99 f_{2}+\frac{1}{3} f_{5}^{3}+18 f_{5}+\frac{5}{3} f_{8}^{3}+46 f_{8} \\
& \text { s.t. }\left\{\begin{array}{l}
f_{1}+f_{2}+f_{4}=9 \\
f_{5}+f_{8}=8 \\
f_{2}+f_{4} \leq 11 \\
f_{4}+f_{8} \leq 5 \\
10 \geq f_{1} \geq 0,8 \geq f_{2} \geq 0,6 \geq f_{4} \geq 0,9 \geq f_{5} \geq 0,7 \geq f_{8} \geq 0
\end{array}\right.
\end{aligned}
$$

We obtain solution $f^{3}=(1.44,3.64,0,3.92,6.92,0,0,1.08)^{\mathrm{T}} \in A$. For O-D pair $\omega 1=(1,4), \tau_{\omega 1}^{3}=182.20$, and for O-D pair $\omega 2=(3,6), \tau_{\omega 2}^{3}=65.89$.

7) After deleting saturated arc $e_{6}$ of flow $f^{3}$ in the network $\aleph$. For O-D pair $\omega 1=(1,4)$, it is easy to verify that the shortest path is $l_{2}$, whereas for O-D pair $\omega 2=(3,6)$, the shortest path is $l_{5}$. Note that $t_{l_{2}}\left(f^{3}\right)=182.20=\tau_{\omega 1}^{3}=182.20, t_{l_{5}}\left(f^{3}\right)=65.89=\tau_{\omega 2}^{3}=65.89$, thus $S_{\omega 1}^{3}=\varnothing, S_{\omega 2}^{3}=\varnothing$. 
8) Because $S^{3}=S_{\omega 1}^{3} \cup S_{\omega 2}^{3}=\varnothing$, the equilibrium flow is $f^{3}=(1.44,3.64,0,3.92,6.92,0,0,1.08)^{\mathrm{T}}$, hence stop.

Note that

$$
\begin{aligned}
& \int_{0}^{f_{e_{1}}} t_{e_{1}}(x) \mathrm{d} x=\int_{0}^{f_{e_{1}}}\left(4 x^{2}+17\right) \mathrm{d} x=\frac{4}{3} f_{e_{1}}^{3}+17 f_{e_{1}}=\frac{4}{3}\left(f_{3}+f_{4}\right)^{3}+17\left(f_{3}+f_{4}\right), \\
& \int_{0}^{f_{e_{2}}} t_{e_{2}}(x) \mathrm{d} x=\int_{0}^{f_{e_{2}}}\left(3 x^{2}+18\right) \mathrm{d} x=f_{e_{2}}^{3}+18 f_{e_{2}}=\left(f_{7}+f_{8}\right)^{3}+18\left(f_{7}+f_{8}\right), \\
& \int_{0}^{f_{e_{3}}} t_{e_{3}}(x) \mathrm{d} x=\int_{0}^{f_{e_{3}}}\left(30 x^{2}+120\right) \mathrm{d} x=10 f_{e_{3}}^{3}+120 f_{e_{3}}=10 f_{1}^{3}+120 f_{1}, \\
& \int_{0}^{f_{e_{4}}} t_{e_{4}}(x) \mathrm{d} x=\int_{0}^{f_{e_{4}}}\left(2 x^{2}+84\right) \mathrm{d} x=\frac{2}{3} f_{e_{4}}^{3}+84 f_{e_{4}}=\frac{2}{3} f_{2}^{3}+84 f_{2}, \\
& \int_{0}^{f_{e_{5}}} t_{e_{5}}(x) \mathrm{d} x=\int_{0}^{f_{e_{5}}}\left(x^{2}+112\right) \mathrm{d} x=\frac{1}{3} f_{e_{5}}^{3}+112 f_{e_{5}}=\frac{1}{3} f_{3}^{3}+112 f_{3}, \\
& \int_{0}^{f_{e_{6}}} t_{e_{6}}(x) \mathrm{d} x=\int_{0}^{f_{e_{6}}}(2 x+18) \mathrm{d} x=f_{e_{6}}^{2}+18 f_{e_{6}}=\left(f_{4}+f_{8}\right)^{2}+18\left(f_{4}+f_{8}\right), \\
& \int_{0}^{f_{e_{7}}} t_{e_{7}}(x) \mathrm{d} x=\int_{0}^{f_{e_{7}}}\left(8 x^{2}+62\right) \mathrm{d} x=\frac{8}{3} f_{e_{7}}^{3}+62 f_{e_{7}}=\frac{8}{3} f_{6}^{3}+62 f_{6}, \\
& \int_{0}^{f_{e_{8}}} t_{e_{8}}(x) \mathrm{d} x=\int_{0}^{f_{e_{8}}}\left(6 x^{2}+65\right) \mathrm{d} x=2 f_{e_{8}}^{3}+65 f_{e_{8}}=2 f_{7}^{3}+65 f_{7}, \\
& \int_{0}^{f_{e_{9}}} t_{e_{9}}(x) \mathrm{d} x=\int_{0}^{f_{e_{9}}}\left(x^{2}+18\right) \mathrm{d} x=\frac{1}{3} f_{e_{9}}^{3}+18 f_{e_{9}}=\frac{1}{3} f_{5}^{3}+18 f_{5}, \\
& \int_{0}^{f_{e_{10}}} t_{e_{10}}(x) \mathrm{d} x=\int_{0}^{f_{e_{10}}}\left(x^{2}+15\right) \mathrm{d} x=\frac{1}{3} f_{e_{10}}^{3}+15 f_{e_{10}}=\frac{1}{3}\left(f_{2}+f_{4}\right)^{3}+15\left(f_{2}+f_{4}\right), \\
& \int_{0}^{f_{e_{11}}} t_{e_{11}}(x) \mathrm{d} x=\int_{0}^{f_{e_{11}}}\left(2 x^{2}+10\right) \mathrm{d} x=\frac{2}{3} f_{e_{11}}^{3}+10 f_{e_{11}}=\frac{2}{3}\left(f_{6}+f_{8}\right)^{3}+10\left(f_{6}+f_{8}\right) .
\end{aligned}
$$

Thus the generalization of Beckmann's formula $Q$ is:

$$
\begin{aligned}
& \operatorname{Min} z(f)=\frac{4}{3}\left(f_{3}+f_{4}\right)^{3}+17\left(f_{3}+f_{4}\right)+\left(f_{7}+f_{8}\right)^{3}+18\left(f_{7}+f_{8}\right)+10 f_{1}^{3}+120 f_{1} \\
& +\frac{2}{3} f_{2}^{3}+84 f_{2}+\frac{1}{3} f_{3}^{3}+112 f_{3}+\left(f_{4}+f_{8}\right)^{2}+18\left(f_{4}+f_{8}\right)+\frac{8}{3} f_{6}^{3}+62 f_{6} \\
& +2 f_{7}^{3}+65 f_{7}+\frac{1}{3} f_{5}^{3}+18 f_{5}+\frac{1}{3}\left(f_{2}+f_{4}\right)^{3}+15\left(f_{2}+f_{4}\right)+\frac{2}{3}\left(f_{6}+f_{8}\right)^{3}+10\left(f_{6}+f_{8}\right) \\
& \text { s.t. }\left\{\begin{array}{l}
f_{1}+f_{2}+f_{3}+f_{4}=9 \\
f_{5}+f_{6}+f_{7}+f_{8}=8 \\
f_{2}+f_{4} \leq 11 \\
f_{3}+f_{4} \leq 6 \\
f_{6}+f_{8} \leq 7 \\
f_{7}+f_{8} \leq 7 \\
f_{4}+f_{8} \leq 5 \\
10 \geq f_{1} \geq 0,8 \geq f_{2} \geq 0,6 \geq f_{3} \geq 0,5 \geq f_{4} \geq 0, \\
9 \geq f_{5} \geq 0,7 \geq f_{6} \geq 0,7 \geq f_{7} \geq 0,5 \geq f_{8} \geq 0 .
\end{array}\right.
\end{aligned}
$$

It is easy to verify that $f=(1.44,3.64,0,3.92,6.92,0,0,1.08)^{\mathrm{T}}$ is the solution of the generalization of Beckmann's formula $Q(\operatorname{Min} z(f)=1327.31)$. Clearly, $f$ is an equilibrium flow for the TEPCCA.

Note that $g=(1.46,3.74,0,3.80,6.80,0,0,1.20)^{\mathrm{T}}$ is also an equilibrium flow for the TEPCCA, but it is not a solution of the generalization of Beckmann's formula Q, i.e., Theorem 3.1 is a sufficient condition only, not a necessary condition. 


\section{Acknowledgements}

This work was supported by National Natural Science Foundation of China (Grant No. 11271389).

\section{References}

[1] Wardrop, J. (1952) Some Theoretical Aspects of Road Traffic Research. Proceedings of the Institute of Civil Engineers, Part II, 1, 325-378. http://dx.doi.org/10.1680/ipeds.1952.11362

[2] Beckmann, M.J., McGuire, C.B. and Winsten, C.B. (1956) Studies in the Economics of Transportation. Yale University Press, New Haven.

[3] Chen, G.Y. and Yen, N.D. (1993) On the Variational Inequality Model for Network Equilibrium [Internal Report 3. 196 (724)]. Department of Mathematics, University of Pisa.

[4] Lin, Z. (2010) The Study of Traffic Equilibrium Problems with Capacity Constraints of Arcs. Nonlinear Analysis: Real World Applications, 11, 2280-2284. http://dx.doi.org/10.1016/j.nonrwa.2009.07.002

[5] Lin, Z. (2010) On Existence of Vector Equilibrium Flows with Capacity Constraints of Arcs. Nonlinear Analysis, 72, 2076-2079. http://dx.doi.org/10.1016/j.na.2009.10.007

[6] Xu, Y.D. and Li, S.J. (2014) Vector Network Equilibrium Problems with Capacity Constraints of Arcs and Nonlinear Scalarization Methods. Applicable Analysis: An International Journal, 93, 2199-2210. http://dx.doi.org/10.1080/00036811.2013.875160

[7] Chiou, S.W. (2010) An Efficient Algorithm for Computing Traffic Equilibria Using TRANSYT Model. Applied Mathematical Modelling, 34, 3390-3399. http://dx.doi.org/10.1016/j.apm.2010.02.028

[8] Xu, M., Chen, A., Qu, Y. and Gao, Z. (2011) A Semismooth Newton Method for Traffic Equilibrium Problem with a General Nonadditive Route Cost. Applied Mathematical Modelling, 35, 3048-3062. http://dx.doi.org/10.1016/j.apm.2010.12.021

[9] Chen, A., Zhou, Z. and Xu, X.D. (2012) A Self-Adaptive Gradient Projection Algorithm for the Nonadditive Traffic Equilibrium Problem. Computers \& Operations Research, 39, 127-138. http://dx.doi.org/10.1016/j.cor.2011.02.018 